\title{
SCIENTIFIC REPORTS

\section{OPEN A scoring system to predict the occurrence of very late stent thrombosis following percutaneous coronary intervention for acute coronary syndrome}

\author{
Xiang Wang ${ }^{1,5}$, Xinxin Chen ${ }^{1,5}$, Tao Tian ${ }^{1}$, Hongzhao You ${ }^{2}$, Yulin $\mathrm{Li}^{3}$, Muli Wu ${ }^{4}$, Xiaoyu Du ${ }^{1}$, \\ $\mathrm{He} \mathrm{Cail}^{1}$, Yang Zheng ${ }^{1 *} \&$ Jie Du ${ }^{3 *}$
}

We aimed to derive and validate an effective risk score to identify high-risk patients of very late stent thrombosis (VLST), following percutaneous coronary intervention (PCI) for acute coronary syndrome (ACS). Stepwise multivariable Cox regression was used to build the risk model using data from 5,185 consecutive ACS patients treated with $\mathrm{PCl}$ (derivation cohort) and 2,058 patients from the external validation cohort. Eight variables were independently associated with the development of VLST: history of diabetes mellitus, previous $\mathrm{PCl}$, acute myocardial infarction as admitting diagnosis, estimated glomerular filtration rate $<90 \mathrm{ml} / \mathrm{min} / 1.73 \mathrm{~m}^{2}$, three-vessel disease, number of stents per lesion, sirolimus-eluting stent, and no post-dilation. Based on the derived score, patients were classified into low- $(\leq 7)$, intermediate- $(8-9)$, and high- $(\geq 10)$ risk categories. Observed VLST rates were $0.5 \%$, $2.2 \%$, and $8.7 \%$ and $0.45 \%, 2.3 \%$, and $9.3 \%$ across the 3 risk categories in the derivation and validation cohorts, respectively. High discrimination (c-statistic $=0.80$ and 0.82 in the derivation and validation cohorts, respectively) and excellent calibration were observed in both cohorts. VLST risk score, a readily useable and efficient tool to identify high-risk patients of VLST after PCI for ACS, may aid in riskstratification and pre-emptive decision-making.

Percutaneous coronary intervention (PCI) is currently the primary treatment for acute coronary syndrome (ACS), which is one of the most serious threats to human health worldwide. Very late stent thrombosis (VLST), defined as thrombosis that occurs more than 1 year after stent implantation, is a catastrophic complication of PCI and accounts for $20 \%$ of new cases of myocardial infarction (MI) after index PCI ${ }^{1}$. The adjusted mortality is 4 -fold higher for VLST than that for MI, which is not related to a previously stented site ${ }^{1}$. Tens of millions of patients worldwide have received stent implants in the past decade, and registry reports show evidence of increased VLST risk up to 5 years postoperatively, with no clear evidence of attenuation despite stent iteration and recent progress in PCI technology2 ${ }^{2}$. Therefore, early identification of high-risk patients with VLST is of great importance as this could inform tailored management regiens, including intensive monitoring and intervention, which may improve patient outcomes. However, owing to the existence of confounding factors, traditional single-factor risk analysis cannot adequately identify high-risk patients at an individual level. In 2012, Dangas et al. developed a risk-score system for late stent thrombosis (the LST risk score), and this proved useful in predicting the development of VLST. However, the predictive ability of the score is limited (c-statistic $=0.66)^{3}$. There is currently no verified clinical risk score for the prediction of VLST.

\footnotetext{
${ }^{1}$ Department of Cardiovascular Disease Center, First Hospital of Jilin University, Jilin University, Jilin, China. ${ }^{2}$ State Key Laboratory of Cardiovascular Disease, Cardiovascular Institute, Fuwai Hospital and National Centre for Cardiovascular Diseases, Chinese academy of Medical Sciences \& Peking Union Medical College, Beijing, China. ${ }^{3}$ Beijing Institute of Heart, Lung and Blood Vessel Diseases, Beijing Anzhen Hospital, Capital Medical University, Beijing, China. ${ }^{4}$ Department of Cardiovascular Center, First Affiliated Hospital of Shantou University Medical College, Shantou University, Guangdong, China. ${ }^{5}$ These authors contributed equally: Xiang Wang and Xinxin Chen. *email: wangxiang17@mails.jlu.edu.cn; jiedu@ccmu.edu.cn
} 
In the present study, we aimed to develop a risk score for VLST based on clinical variables from a large cohort of consecutive patients with ACS and independently validate the system in another cohort with the aim of identifying patients at high risk of VLST.

\section{Results}

Patient characteristics. A total of 6,038 patients were eligible for the derivation cohort; of which, 236 were excluded owing to death prior to discharge, 413 subjects refused to participate in follow-up interviews, and 204 were lost to follow-up. Finally, 5,185 patients were enrolled (average age $59.85 \pm 9.95$ years, $68.60 \%$ male). The median length of follow-up was 1,502 days.

For the validation cohort, of the 2,438 patients identified as eligible, we excluded 88 patients who died prior to discharge, 186 who did not agree to participate in follow-up interviews, and 106 who were lost to follow-up. Finally, 2,058 patients were enrolled (average age $60.00 \pm 10.22$ years, $68.71 \%$ male). The median length of follow-up was 1,422 days. Supplementary Table S1 provides a comparison of the baseline and procedural characteristics as well as laboratory data between patients within the two cohorts.

The median time from index PCI to the occurrence of VLST was 697 and 803 days for the derivation and validation cohorts, respectively. The incidence of VLST was not significantly different between the derivation and validation cohorts at $1.77 \%$ and $1.70 \%$, respectively $(\mathrm{P}=0.829)$. Table 1 provides a comparison of the baseline and procedural characteristics as well as laboratory data between patients with and without VLST in the derivation cohort. A significantly larger number of patients were admitted with an acute MI among those with VLST $(\mathrm{P}<0.001)$, and the peak troponin I level was higher among these patients; although, the difference was not statistically significant $(\mathrm{P}=0.051)$. Patients with VLST experienced significantly more Thrombolysis in Myocardial Infarction (TIMI) flow grade 0 before PCI $(\mathrm{P}=0.017)$, stent overlap was significantly higher $(\mathrm{P}=0.013)$, and stent length significantly lon ger $(P=0.001)$ during $P C I$ than that among subjects without VLST. There was no difference between the occurrence of slow flow $(\mathrm{P}=0.619)$ and no reflow $(\mathrm{P}=0.547)$ after PCI between patients with VLST and those without. More patients without VLST received dual-antiplatelet therapy (DAPT) during follow-up compar ed to those that received DAPT with VLST; although, this was not significantly different (44.37\% vs. $38.04 \%$, respectively, $\mathrm{P}=0.226)$.

Development of the very late stent thrombosis risk score. In total, 12 predictors were identified for the development of VLST from the univariable analysis in the derivation cohort, and 4 independent risk factors were identified in the literature (Supplementary Table S2). Eight predictors were independently associated with the occurrence of VLST by multivariable Cox regression analysis as follows: history of diabetes mellitus (DM), previous PCI, acute MI as the admitting diagnosis, estimated glomerular filtration rate (eGFR) of $<90 \mathrm{ml} /$ $\mathrm{min} / 1.73 \mathrm{~m}^{2}$, three-vessel disease, number of stents per lesion, sirolimus-eluting stent (SES), and no post-dilation. The variance-inflation factor was $<2$, indicating the absence of multicollinearity among variables in the model. The $\mathrm{p}$-value from the Hosmer-Lemeshow goodness-of-fit test of the final model was 0.082 for the derivation cohort. The c-statistic of the final model was 0.81 (95\% confidence interval [CI]: 0.76-0.86), and cross-validation predicted a slight decrease in discriminative ability (c-statistic $0.75,95 \%$ CI: $0.70-0.80$ ).

The points assigned to each variable of the risk-scoring system are shown in Table 2. The relationship between score value and the predicted incidence of VLST is illustrated in Supplementary Fig. S1 $(\mathrm{P}=0.345$ from the Hosmer-Lemeshow test of the score). Supplementary Fig. S2 presents the calibration plot of predicted vs. observed incidence of VLST development among the derivation cohort across deciles of the risk score, which shows excellent calibration. The c-statistic and hazard ratio (HR) of the score was 0.80 (95\% CI: $0.75-0.85)$ and 1.69 (95\% CI: 1.54-1.85), respectively, in the derivation cohort. Levels of risk were defined according to the predicted incidence of VLST: low risk $=$ score of $\leq 7$, corresponding to a predicted incidence of $<1 \%(3,135$ patients, $60.5 \%$ of the derivation cohort); intermediate risk $=8$ or 9 points, corresponding to a predicted incidence of $1 \%-3 \%$ ( 1,837 patients, $35.4 \%$ of the derivation cohort); high-risk was $\geq 10$ points, corresponding to a predicted incidence of $>3 \%$ ( 473 patients, $9.1 \%$ of the derivation cohort). The observed incidences of VLST according to these cut-offs were $0.5 \%, 2.2 \%$, and $8.7 \%$, respectively, in the derivation cohort (Fig. 1 ).

Risk-score validation. The c-statistic of the final model was 0.83 (95\% CI: 0.78-0.89) in the validation cohort, with a p-value of 0.66 from the Hosmer-Lemeshow test. The c-statistic of the VLST risk score was 0.82 (95\% CI: 0.76-0.90), with a p-value of 0.97 from the Hosmer-Lemeshow test. The HR of the risk score was 1.74 for the validation cohort (95\% CI: 1.53-1.99). The predicted and observed incidences of VLST across deciles of the risk score in the validation cohort are illustrated in Supplementary Fig. S2. According to predicted risk, 64.67\% $(\mathrm{n}=1,331), 26.97 \%(\mathrm{n}=555)$, and $8.36 \%(\mathrm{n}=172)$ of the validation cohort were identified as low, intermediate, and high risk, respectively. The observed incidences of VLST in each risk group were $0.45 \%, 2.34 \%$, and $9.3 \%$, respectively, in the validation cohort (Fig. 1). The risk scores and the occurrence of VLST in the derivation and validation cohorts are depicted in Supplementary Fig. S3.

The prediction model was further evaluated by decision-curve analysis (Fig. 2). The results demonstrated that, for relevant decision thresholds, the VLST risk-score model provided a substantial net clinical benefit compared with that of the LST risk-score model, which included only three variables (current smoking, history of PCI, and ST-elevated MI [STEMI] as the admitting diagnosis). For a decision threshold of $2 \%$ of VLST risk, compared with the LS risk score, the VLST risk score should identify 4 additional cases, without any false-positive results, from a population of 1,000 patients with a $1.7 \%$ incidence of VLST. 


\begin{tabular}{|c|c|c|c|}
\hline Variables & $\begin{array}{l}\text { Patients without } \\
\text { VLST }(n=5093)\end{array}$ & $\begin{array}{l}\text { Patients with } \\
\text { VLST }(\mathrm{n}=92)\end{array}$ & p-value \\
\hline Age (years) & $59.83 \pm 9.93$ & $60.71 \pm 11.33$ & 0.211 \\
\hline Male sex & $3489(68.51)$ & $68(73.91)$ & 0.268 \\
\hline Diabetes Mellitus & $1293(25.39)$ & $36(39.13)$ & 0.003 \\
\hline Hypertension & $2670(52.42)$ & $51(55.43)$ & 0.567 \\
\hline Dyslipidaemia & $1612(31.65)$ & $28(30.43)$ & 0.804 \\
\hline Current smoker & $2860(56.16)$ & $54(58.70)$ & 0.626 \\
\hline History of drinking & $751(14.75)$ & $13(14.13)$ & 0.869 \\
\hline History of stroke/TIA & $367(7.21)$ & $12(13.04)$ & 0.033 \\
\hline History of PVD & $86(1.69)$ & $2(2.17)$ & 0.670 \\
\hline History of heart failure & $135(2.65)$ & $2(2.17)$ & 0.559 \\
\hline Previous CABG & $140(2.75)$ & $4(4.35)$ & 0.325 \\
\hline Previous PCI & $94(1.85)$ & $13(14.13)$ & $<0.001$ \\
\hline Previous MI & $347(6.81)$ & $10(10.87)$ & 0.128 \\
\hline AMI as admitting diagnosis & $3046(59.81)$ & $72(78.26)$ & $<0.001$ \\
\hline Cardiogenic shock & $110(2.16)$ & $3(3.26)$ & 0.454 \\
\hline Three-vessel disease & $1422(27.92)$ & $42(45.65)$ & $<0.001$ \\
\hline Chronic total occlusion & $235(4.61)$ & $4(4.35)$ & 0.580 \\
\hline Moderate to severe calcification & $182(3.57)$ & $3(3.26)$ & 0.583 \\
\hline Moderate to severe tortuosity & $59(1.16)$ & $1(1.09)$ & 0.712 \\
\hline Ostial lesion & $117(2.30)$ & $2(2.17)$ & 0.646 \\
\hline Proximal lesion & $3481(68.35)$ & $63(68.48)$ & 0.979 \\
\hline Bifurcation lesion & $621(12.19)$ & $17(18.48)$ & 0.069 \\
\hline Visual thrombus & $588(11.55)$ & $10(10.87)$ & 0.841 \\
\hline Coronary aneurysm & $27(0.53)$ & $1(1.09)$ & 0.395 \\
\hline Vessel ectasia & $57(1.11)$ & $2(2.17)$ & 0.282 \\
\hline Vessel ulceration & $38(0.75)$ & $1(1.09)$ & 0.504 \\
\hline Vessel dissection & $52(1.02)$ & $2(2.17)$ & 0.249 \\
\hline \multicolumn{4}{|l|}{ Culprit vessel } \\
\hline LM & $103(2.02)$ & $2(2.17)$ & 0.710 \\
\hline LAD & $2682(52.66)$ & $47(51.09)$ & 0.764 \\
\hline LCX & $879(17.26)$ & $17(18.48)$ & 0.759 \\
\hline $\mathrm{RCA}$ & $1689(33.16)$ & $32(34.78)$ & 0.744 \\
\hline TIMI flow grade 0 before PCI & $1668(32.75)$ & $41(44.57)$ & 0.017 \\
\hline Thrombus aspiration & $654(12.84)$ & $10(10.87)$ & 0.575 \\
\hline Slow flow after PCI & $173(3.40)$ & $3(3.26)$ & 0.619 \\
\hline No reflow after PCI & $43(0.84)$ & $1(1.08)$ & 0.547 \\
\hline \multicolumn{4}{|l|}{ Stent type used during PCI } \\
\hline SES & $3502(68.76)$ & $76(82.61)$ & 0.004 \\
\hline ZES & $889(17.46)$ & $9(9.78)$ & 0.054 \\
\hline EES & $702(13.78)$ & $7(7.61)$ & 0.088 \\
\hline Stent overlap & $968(19.01)$ & $27(29.35)$ & 0.013 \\
\hline No post-dilation & $2496(49.01)$ & $14(15.22)$ & $<0.001$ \\
\hline Reference vessel diameter (mm) & $3.03 \pm 0.40$ & $2.99 \pm 0.36$ & 0.541 \\
\hline Min-stent diameter $(\mathrm{mm})$ & $2.99 \pm 0.41$ & $2.92 \pm 0.39$ & 0.117 \\
\hline Max-stent diameter $(\mathrm{mm})$ & $3.06 \pm 0.41$ & $3.05 \pm 0.38$ & 0.826 \\
\hline Total stent length $(\mathrm{mm})$ & $31.70 \pm 15.62$ & $38.46 \pm 20.71$ & 0.001 \\
\hline Stent release pressure (atm) & $13.95 \pm 2.99$ & $13.93 \pm 2.80$ & 0.901 \\
\hline No. of stents per lesion & $1.21 \pm 0.46$ & $1.42 \pm 0.65$ & $<0.001$ \\
\hline LVEF & $55.15 \pm 4.52$ & $54.55 \pm 3.68$ & 0.033 \\
\hline Peak troponin I & $37.37 \pm 61.64$ & $40.55 \pm 72.25$ & 0.051 \\
\hline eGFR $<90\left(\mathrm{ml} / \mathrm{min} / 1.73 \mathrm{~m}^{2}\right)$ & $860(16.89)$ & $32(34.78)$ & $<0.001$ \\
\hline $\mathrm{WBC}\left(* 10^{9} / \mathrm{l}\right)$ & $8.75 \pm 3.24$ & $12.49 \pm 3.92$ & $<0.001$ \\
\hline $\operatorname{HGB}(\mathrm{g} / \mathrm{l})$ & $141.64 \pm 16.22$ & $144.53 \pm 13.08$ & 0.171 \\
\hline Platelet $\left(* 10^{9} / 1\right)$ & $225.31 \pm 62.49$ & $224.24 \pm 56.09$ & 0.816 \\
\hline TCL $(\mathrm{mmol} / \mathrm{l})$ & $4.57 \pm 1.05$ & $4.69 \pm 1.31$ & 0.756 \\
\hline $\mathrm{LDL}(\mathrm{mmol} / \mathrm{l})$ & $2.86 \pm 0.81$ & $3.05 \pm 1.08$ & 0.327 \\
\hline
\end{tabular}




\begin{tabular}{|l|l|l|l|}
\hline Variables & $\begin{array}{l}\text { Patients without } \\
\text { VLST }(\mathbf{n = 5 0 9 3})\end{array}$ & $\begin{array}{l}\text { Patients with } \\
\text { VLST }(\mathbf{n = 9 2})\end{array}$ & p-value \\
\hline HDL $(\mathrm{mmol} / \mathrm{l})$ & $1.13 \pm 0.28$ & $1.13 \pm 0.35$ & 0.672 \\
\hline TG $(\mathrm{mmol} / \mathrm{l})$ & $2.12 \pm 1.39$ & $2.32 \pm 1.71$ & 0.558 \\
\hline Fasting blood-glucose $(\mathrm{mmol} / \mathrm{l})$ & $6.70 \pm 2.73$ & $7.82 \pm 3.98$ & 0.025 \\
\hline HbAlc $(\%)$ & $6.27 \pm 1.53$ & $5.80 \pm 1.61$ & $<0.001$ \\
\hline Fibrinogen $(\mathrm{g} / \mathrm{l})$ & $3.17 \pm 0.88$ & $3.37 \pm 1.01$ & 0.012 \\
\hline DAPT & $2260(44.37)$ & $35(38.04)$ & 0.226 \\
\hline
\end{tabular}

Table 1. Baseline, procedural characteristics, and laboratory test results of patients with and without VLST in the derivation cohort. AMI, acute myocardial infarction; CABG, coronary artery bypass graft; DAPT, dual-antiplatelet therapy; EES, everolimus eluting stent; eGFR, estimated glomerular filtration rate; HDL, high density lipoprotein; HGB, haemoglobin; LAD, left anterior descending artery; LCX, left circumflex artery; LDL, low density lipoprotein; LM, left main; LVEF, left ventricular eject fraction; MI, myocardial infarction; PCI, percutaneous coronary intervention; PVD, peripheral vascular disease; RCA, right coronary artery; SES, sirolimus-eluting stent; TCL, total cholesterol; TG, triglyceride; TIA, transient ischemic attack; TIMI, Thrombolysis in Myocardial Infarction; VLST, very late stent thrombosis; WBC, white blood cell; ZES, zotarolimus eluting stent

\begin{tabular}{|l|l|l|l|l|}
\hline Variables & Corrected $\boldsymbol{\beta}$ & $\begin{array}{l}\text { Multivariable HR } \\
(\mathbf{9 5} \mathbf{C I})\end{array}$ & p-value & $\begin{array}{l}\text { Risk score } \\
\text { assigned weight }\end{array}$ \\
\hline Diabetes Mellitus & 0.58 & $1.78(1.17-2.72)$ & 0.008 & 1 \\
\hline Previous PCI & 1.68 & $5.34(2.72-10.45)$ & $<0.001$ & 3 \\
\hline AMI as admitting diagnosis & 0.54 & $1.71(1.07-2.72)$ & 0.024 & 1 \\
\hline eGFR $<90\left(\mathrm{ml} / \mathrm{min} / 1.73 \mathrm{~m}^{2}\right)$ & 0.76 & $2.13(1.38-3.29)$ & 0.001 & 1 \\
\hline 3-vessel disease & 0.51 & $1.66(1.09-2.53)$ & 0.019 & 1 \\
\hline No. of stents per lesion & 0.96 & $2.62(1.90-3.61)$ & $<0.001$ & 2 No. \\
\hline Stent type-SES & 0.66 & $1.94(1.13-3.34)$ & 0.017 & 1 \\
\hline No post-dilation & 1.93 & $0.145(0.08-0.26)$ & $<0.001$ & 4 \\
\hline
\end{tabular}

Table 2. Multivariable predictors of VLST in the derivation cohort and their respective weights in the VLST risk score. AMI, acute myocardial infarction; eGFR, estimated glomerular filtration rate; No., number; PCI, percutaneous coronary intervention; SES, sirolimus-eluting stent. VLST, very late stent thrombosis.
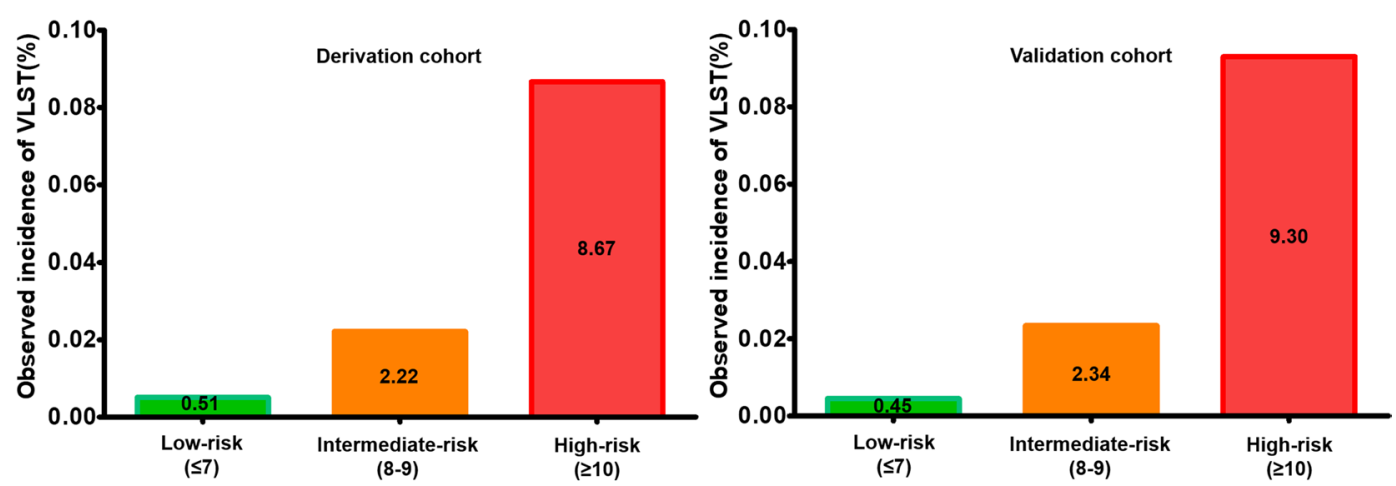

Figure 1. Observed incidence of VLST. Observed incidence of VLST according to the categories of the VLST risk score in the derivation and validation cohorts. VLST, very late stent thrombosis.

\section{Discussion}

In this study, development of VLST was independently associated with the following eight variables: history of DM, previous PCI, acute myocardial infarction as admitting diagnosis, estimated glomerular filtration rate $(\mathrm{eGFR})<90 \mathrm{ml} / \mathrm{min} / 1.73 \mathrm{~m}^{2}$, three-vessel disease, number of stents per lesion, SES, and no post-dilation. The observed VLST rates were $0.5 \%, 2.2 \%$, and $8.7 \%$ in the derivation cohort and $0.45 \%, 2.3 \%$, and $9.3 \%$ in the validation cohort across the low-, intermediate-, and high-risk categories, respectively. High discrimination (c-statistic of 0.80 and 0.82 in the derivation and validation cohorts, respectively) and excellent calibration were noted in both cohorts. To the best of our knowledge, this is the first study to provide a simple but useable scoring system for identifying impending VLST after PCI from a large cohort of consecutive patients with ACS. The VLST risk score ( Fig. 3) showed good predictive ability and calibration across the two cohorts. Being based on simple 


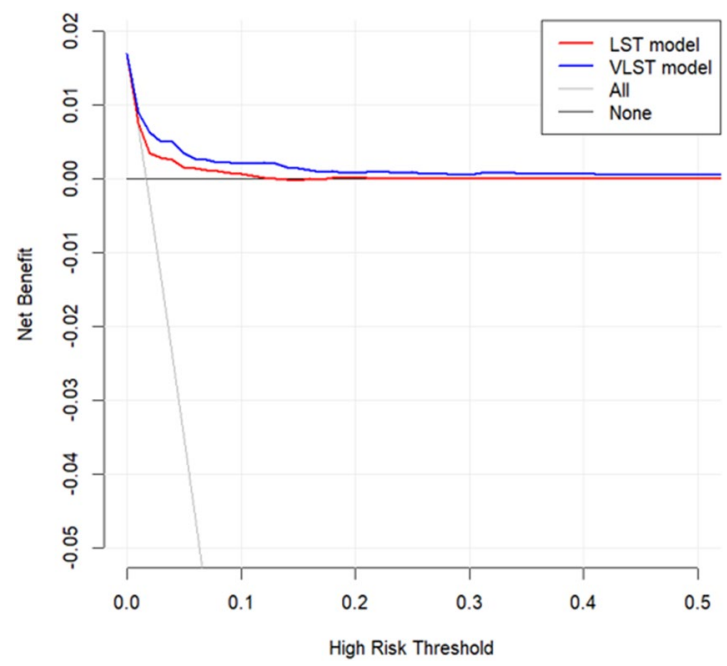

Figure 2. Decision-curve analysis. Net benefit of using a model to predict VLST compared with strategies of 'assuming all' or 'assuming none' patients would be at high-risk for different decision thresholds is shown. The VLST risk score (blue) shows improved benefit compared with the LST risk score (red). LST, late stent thrombosis; VLST, very late stent thrombosis.

\begin{tabular}{|c|c|c|c|c|}
\hline Variables & Point & Total Score & Risk Stratification & Predicted Incidence \\
\hline History of DM & 1 & \multirow{3}{*}{$\leqslant 7$} & \multirow{3}{*}{ Low risk } & \multirow{3}{*}{$<1 \%$} \\
\hline Previous $\mathrm{PCl}$ & 3 & & & \\
\hline AMI as admitting diagnosis & 1 & & & \\
\hline eGFR $<90 \mathrm{ml} / \mathrm{min} / 1.73 \mathrm{~m}^{2}$ & 1 & \multirow{2}{*}{$8-9$} & \multirow{2}{*}{ Intermediate risk } & \multirow{2}{*}{$1-3 \%$} \\
\hline Three vessel disease & 1 & & & \\
\hline Sirolimus-eluting stent & 1 & \multirow{3}{*}{$\geqslant 10$} & \multirow{3}{*}{ High risk } & \multirow{3}{*}{$>3 \%$} \\
\hline No. of stents per lesion & $2 * \mathrm{No}$ & & & \\
\hline No post-dilation & 4 & & & \\
\hline
\end{tabular}

Figure 3. The VLST scoring system. AMI, acute myocardial infarction; DM, diabetes mellitus; eGFR, estimated glomerular filtration rate; PCI, percutaneous coronary intervention; VLST, very late stent thrombosis.

categorical variables, the score could be used to identify high-risk patients, with particular utility in the decision of treatment plans for patients more than 1 year after PCI.

Previous clinical studies have divided the factors that are correlated with the development of VLST into three categories: patient- and lesion-related factors ${ }^{4-6}$, procedural- and stent-related factors ${ }^{7-11}$, and pharmacotherapy-related factors ${ }^{12,13}$. In the present study, five variables were patient- and lesion-related and three were procedural- or stent-related, consistent with previous literature reports ${ }^{4-13}$. The effect of discontinuation of DAPT, which has been suggested to affect the development of VLST during follow-up ${ }^{14}$, had no statistically significant effect on the incidence of VLST in the derivation cohort.

Patient- and lesion-related variables included a history of DM, previous PCI, acute MI as the admitting diagnosis, eGFR $<90 \mathrm{ml} / \mathrm{min} / 1.73 \mathrm{~m}^{2}$, and three-vessel disease. Previous studies have suggested that DM plays an important role in promoting atherosclerosis, and in-stent neoatherosclerosis has been found to be an important underlying mechanism of VLST ${ }^{15,16}$. Additionally, a history of DM has been described as a powerful predictor of subacute, late, and very late stent thrombosis ${ }^{17}$. Previous PCI might indicate prior stent placement and has been included as a variable in the LST risk score ${ }^{3}$. Previous studies have reported higher rates of stent thrombosis (ST) among patients with a history of MI, which might be related to the different plaque characteristics underlying the $\mathrm{MI}$ and angina pectoris ${ }^{18-20}$. Chronic kidney disease could promote the progression of arteriosclerosis and has been found to be an independent risk factor for $\mathrm{ST}^{21,22}$. The three-vessel disease is an indicator of the severity and diffusion of lesions and is correlated with the development of $\mathrm{ST}^{6}$.

Regarding the procedure- and stent-related variables, a greater number of stents indicates longer lesions and stents; thus, more stent overlap, all of which could contribute to the development of ST ${ }^{6}$. Furthermore, no post-dilation was reported as an independent risk factor for VLST in the case of bioresorbable stents ${ }^{23}$, and might lead to stent malposition, which is an important mechanism underlying VLST development ${ }^{7,24,25}$. The representativeantiproliferation-drug-eluting stents of the first-generation drug-eluting stents (DESs) are SESs; local toxicity and inflammatory reactions after implantation of these stents could lead to delayed endothelialisation or even positive remodelling of the vessel walls. This is also an important mechanism involved in the development of VLST ${ }^{16,26-30}$. 
In clinical practice, VLST often occurs more than 1 year after the initial stent placement; therefore, identifying patients at high risk for VLST is extremely important as it may enable the implementation of intensive follow-up and preventative interventions. The LST risk score was established in a cohort of selected patients with ACS and has proven to be equally useful in predicting the occurrence of VLST. The system includes three variables as follows: current smoker, history of previous PCI, and STEMI as the admission diagnosis, but the predictive ability of the score is limited $(c \text {-statistic }=0.66)^{3}$. A recent study ${ }^{31}$ presents a new risk score developed from a small sample case-control study; however, no validation data were reported for this system, and some variables, such as suboptimal stent result, that were included in the score cannot be assessed objectively. Compared with the above study ${ }^{3,31}$, the strength of our study is the inclusion of a cohort of consecutive patients with ACS, which can be expected to reflect real-world clinical practice, and improved discrimination was shown in both the derivation and validation cohorts.

The VLST risk score provides a simple but efficient tool to identify patients at high risk for VLST after PCI. Intensive follow-up should be carried out for these patients, and more aggressive therapy might be required. The independent risk factors included in the present risk model will be significant in guiding the choice of therapeutic approach. For example, selecting fewer stents, using new-generation DESs, and performing effective post-dilation could reduce the incidence of ST. However, the fact that the VLST risk score presented here was developed and validated in patients with ACS means that its value in patients with stable angina pectoris still needs to be proven.

Part of the inclusion criteria for this study was definite ST (assessed by coronary angiography [CAG]) to ensure consistency of events. This might have resulted in some events being missed, though we tried to reduce this situation. In addition, although a single-centre cohort was used for validation, the reliability of the score warrants validation using multi-centre, large-scale prospective cohorts.

The VLST risk score presented here provides a readily useable and efficient tool to identify patients with ACS who are at high risk of developing VLST after PCI. The system may enable further risk stratification and thus, facilitate pre-emptive clinical decision-making.

\section{Methods}

Study population. This study is registered at ClinicalTrials.gov: NCT03491891. We recruited consecutive patients $\geq 18$ years who were admitted to The First Hospital of Jilin University between January 1, 2014 and June 1, 2015 and underwent PCI for treatment of ACS and who had signed informed consent. The exclusion criteria were as follows: expected survival time of less than 1 year, severe organ failure, malignancy, a surgical plan for important organs within 1 year after PCI and antiplatelet drugs should be discontinued; death prior to discharge; and refusal to sign informed consent or take part in follow-up interviews or patients who were lost to follow-up after discharge.

For the external validation cohort, we recruited all consecutive ACS patients who were admitted to the first affiliated hospital of Shantou University for PCI between January 1, 2014 and June 1, 2015, and the same exclusion criteria were applied.

All patients enrolled were treated with newer or second-generation DESs (Supplementary Table S3) and DAPT, lasting for at least one year. Antiplatelet therapy continued beyond this time was based on the decision of patients' personal doctors considering their specific conditions (Supplementary Table S4).

In order to improve the diagnosis of VLST, we set up a 24-hour online consultation to ensure the patients enrolled would receive professional medical evaluation and medical advice immediately when they have cardiac symptoms. For highly suspicious patients, they will be immediately transferred to the nearby Chest Pain Center, and CAG images of confirmed patients will be uploaded to the core laboratory for analysis.

Ethical information. Ethics committee approval (NO. 2013-256) was obtained from the appropriate committee of both institutes (Medical Ethics Committee of The First Hospital of Jilin University, First Affiliated Hospital of Shantou University Medical College Ethics Committee), and the study was performed in accordance with the ethical standards laid out in the 1964 Declaration of Helsinki and its later amendments. All patients provided written informed consent.

Definitions and endpoints. We defined VLST according to the 2007 criteria of the Academic Research Consortium (ARC) for ST; specifically, ST that occurred more than one year after coronary stent implantation and was confirmed by CAG. All documented cases of VLST were determined by CAG, and the procedures were independently reviewed by two experienced interventional cardiologists. If a consensus could not be reached between the two reviewers, a third reviewer was consulted. The primary endpoint of this study was definite ST.

Statistical analysis. Categorical data are presented as numbers (percentage). Continuous data are presented as mean \pm standard deviation. Categorical data were compared using the chi-square test or Fisher's exact test, while quantitative data were compared using the Mann-Whitney U test. All statistical tests were 2-sided with a 0.05 significance level.

The derivation cohort was used to identify predictors for the occurrence of VLST and to establish a risk-scoring system, which was verified using the validation cohort. Significant variables $(\mathrm{P}<0.05)$ in univariable Cox regression analysis and independent risk factors reported in previous literature were included in the multivariate Cox regression analysis and the optimal predictive variables of VLST were identified using the backward stepwise method. Some variables were excluded, such as leukocyte count (for which there was insufficient evidence to definitively conclude that the increased count was not owing to infection or other factors). Procedural variables, such as stent diameter, length, type, and post-dilation, were based on the operator's subjective assessment during PCI. 
Multicollinearity between the variables in the final model was assessed by calculation of the variance-inflation factor. These corrected coefficients of significant predictors were then divided by the value of the lowest coefficient and rounded to the nearest integer to assign a weight to each predictor. The total score was calculated for each patient by adding these weights together. An objective assessment of calibration was obtained using the Hosmer-Lemeshow goodness-of-fit test and by plotting the observed vs. predicted incidence rates across the risk-score deciles. The predictive performance of the ris model was assessed using the c-statistic and was cross-validated (using the leave-one-out method) in the development cohort. Clinical utility was evaluated using decision-curve analysis. Statistical analyses were performed using the Statistical Package for Social Sciences version 23 (SPSS, Chicago, IL, USA) and R version 3.5.0 (R Foundation for Statistical Computing, Vienna, Austria) with the rms package.

\section{Data availability}

All access data are available at www.figshare.com.

Received: 7 November 2019; Accepted: 31 March 2020;

Published online: 14 April 2020

\section{References}

1. Lemesle, G. et al. Incident myocardial infarction and very late stent thrombosis in outpatients with stable coronary artery disease. J Am Coll Cardiol 69, 2149-2156, https://doi.org/10.1016/j.jacc.2017.02.050 (2017).

2. Daemen, J. et al. Early and late coronary stent thrombosis of sirolimus-eluting and paclitaxel-eluting stents in routine clinical practice: data from a large two-institutional cohort study. The Lancet 369, 667-678, https://doi.org/10.1016/s0140-6736(07)60314-6 (2007).

3. Dangas, G. D. et al. Development and validation of a stent thrombosis risk score in patients with acute coronary syndromes. JACC Cardiovasc Interv 5, 1097-1105, https://doi.org/10.1016/j.jcin.2012.07.012 (2012).

4. van Werkum, J. W. et al. Predictors of coronary stent thrombosis: the Dutch Stent Thrombosis Registry. J Am Coll Cardiol 53, 1399-1409, https://doi.org/10.1016/j.jacc.2008.12.055 (2009).

5. van Boven, N. et al. Stent thrombosis in early-generation drug-eluting stents versus newer-generation everolimus-eluting stent assorted by LVEF. Heart 101, 50-57, https://doi.org/10.1136/heartjnl-2014-305743 (2015).

6. Waksman, R. et al. Correlates and outcomes of late and very late drug-eluting stent thrombosis: results from DESERT (International Drug-Eluting Stent Event Registry of Thrombosis). JACC Cardiovasc Interv 7, 1093-1102, https://doi.org/10.1016/j.jcin.2014.04.017 (2014).

7. Parodi, G. et al. Stent-related defects in patients presenting with stent thrombosis: differences at optical coherence tomography between subacute and late/very late thrombosis in the Mechanism Of Stent. Thrombosis (MOST) study. EuroIntervention 9, 936-944, https://doi.org/10.4244/EIJV9I8A157 (2013).

8. Sarno, G. et al. Stent thrombosis in new-generation drug-eluting stents in patients with STEMI undergoing primary PCI: a report from SCAAR. J Am Coll Cardiol 64, 16-24, https://doi.org/10.1016/j.jacc.2014.04.022 (2014).

9. Philip, F. et al. Stent thrombosis with second-generation drug-eluting stents compared with bare-metal stents: network metaanalysis of primary percutaneous coronary intervention trials in ST-segment-elevation myocardial infarction [corrected]. Circ Cardiovasc Interv 7, 49-61, https://doi.org/10.1161/CIRCINTERVENTIONS.113.000412 (2014).

10. Wykrzykowska, J. J. et al. Bioresorbable scaffolds versus metallic stents in routine PCI. N Engl J Med 376, 2319-2328, https://doi. org/10.1056/NEJMoa1614954 (2017).

11. Nakazawa, G. et al. Coronary responses and differential mechanisms of late stent thrombosis attributed to first-generation sirolimusand paclitaxel-eluting stents. J Am Coll Cardiol 57, 390-398, https://doi.org/10.1016/j.jacc.2010.05.066 (2011).

12. Pesarini, G. et al. Late and very late coronary stent thrombosis: Intravascular ultrasound findings and associations with antiplatelet therapy. Catheter Cardiovasc Interv 82, 1056-1065, https://doi.org/10.1002/ccd.24938 (2013).

13. Huang, K. N., Grandi, S. M., Filion, K. B. \& Eisenberg, M. J. Late and very late stent thrombosis in patients with second-generation drug-eluting stents. Can J Cardiol 29, 1488-1494, https://doi.org/10.1016/j.cjca.2013.04.001 (2013).

14. Loh, J. P. et al. Impact of early versus late clopidogrel discontinuation on stent thrombosis following percutaneous coronary intervention with first- and second-generation drug-eluting stents. Am J Cardiol 113, 1968-1976, https://doi.org/10.1016/j. amjcard.2014.03.041 (2014).

15. Otsuka, F. et al. Neoatherosclerosis: overview of histopathologic findings and implications for intravascular imaging assessment. Eur Heart J36, 2147-2159, https://doi.org/10.1093/eurheartj/ehv205 (2015).

16. Taniwaki, M. et al. Mechanisms of very late drug-eluting stent thrombosis assessed by optical coherence tomography. Circulation 133, 650-660, https://doi.org/10.1161/CIRCULATIONAHA.115.019071 (2016).

17. Dangas, G. D. et al. Frequency and predictors of stent thrombosis after percutaneous coronary intervention in acute myocardial infarction. Circulation 123, 1745-1756, https://doi.org/10.1161/CIRCULATIONAHA.110.981688 (2011).

18. Reed, G. W., Rossi, J. E. \& Cannon, C. P. Acute myocardial infarction. The Lancet 389, 197-210, https://doi.org/10.1016/s01406736(16)30677-8 (2017).

19. Kini, A. S. et al. Fibrous cap thickness by optical coherence tomography in vivo. J Am Coll Cardiol 69, 644-657, https://doi. org/10.1016/j.jacc.2016.10.028 (2017).

20. Heusch, G. \& Gersh, B. J. The pathophysiology of acute myocardial infarction and strategies of protection beyond reperfusion: a continual challenge. Eur Heart J 38, 774-784, https://doi.org/10.1093/eurheartj/ehw224 (2017).

21. Jones, D. A. et al. Contemporary analysis of incidence and outcomes of stent thrombosis presenting as ST elevation myocardial infarction in a primary percutaneous coronary intervention cohort. Am J Cardiol 112, 1347-1354, https://doi.org/10.1016/j. amjcard.2013.07.002 (2013).

22. Ricardo, A. C. et al. Healthy lifestyle and risk of kidney disease progression, atherosclerotic events, and death in CKD: findings from the Chronic Renal Insufficiency Cohort (CRIC) study. American Journal of Kidney Diseases 65, 412-424, https://doi.org/10.1053/j. ajkd.2014.09.016 (2015).

23. Ellis, S. G. et al. Clinical, angiographic, and procedural correlates of acute, subacute, and late absorb scaffold thrombosis. JACC Cardiovasc Interv 10, 1809-1815, https://doi.org/10.1016/j.jcin.2017.06.067 (2017).

24. Kosonen, P. et al. Intravascular ultrasound assessed incomplete stent apposition and stent fracture in stent thrombosis after bare metal versus drug-eluting stent treatment the Nordic Intravascular Ultrasound Study (NIVUS). Int J Cardiol 168, 1010-1016, https://doi.org/10.1016/j.ijcard.2012.10.033 (2013).

25. Souteyrand, G. et al. Mechanisms of stent thrombosis analysed by optical coherence tomography: insights from the national PESTO French registry. Eur Heart J 37, 1208-1216, https://doi.org/10.1093/eurheartj/ehv711 (2016). 
26. De Luca, G. et al. Everolimus eluting stent vs first generation drug-eluting stent in primary angioplasty: A pooled patient-level metaanalysis of randomized trials. Int J Cardiol 244, 121-127, https://doi.org/10.1016/j.ijcard.2017.06.022 (2017).

27. De Luca, G. et al. Time course, predictors and clinical implications of stent thrombosis following primary angioplasty. Insights from the DESERT cooperation. Thromb Haemost 110, 826-833, https://doi.org/10.1160/TH13-02-0092 (2013).

28. Ishihara, T. et al. Very late stent thrombosis 5 years after implantation of a sirolimus-eluting stent observed by angioscopy and optical coherence tomography. JACC Cardiovasc Interv 6, e28-30, https://doi.org/10.1016/j.jcin.2012.12.127 (2013).

29. Adriaenssens, T. et al. Optical coherence tomography findings in patients with coronary stent thrombosis: a report of the PRESTIGE consortium (Prevention of Late Stent Thrombosis by an Interdisciplinary Global European Effort). Circulation 136, 1007-1021, https://doi.org/10.1161/CIRCULATIONAHA.117.026788 (2017).

30. Lee, S.Y. et al. Characteristics of earlier versus delayed presentation of very late drug-eluting stent thrombosis: an optical coherence tomographic study. J Am Heart Assoc 6, https://doi.org/10.1161/JAHA.116.005386 (2017).

31. Godschalk, T. C. et al. A clinical risk score to identify patients at high risk of very late stent thrombosis. J Interv Cardiol 31, 159-169, https://doi.org/10.1111/joic.12494 (2018).

\section{Acknowledgements}

We would like to thank Editage (www.editage.cn) for English language editing. The research was supported by National Key R\&D Program of China (2016YFC0900903 to Y.Z.). The funders had no role in the study design, data collection, data analysis and interpretation, writing of the report, or the decision to submit the article for publication.

\section{Author contributions}

Y. Z., J. D. and X. W. conceived and designed the paper. X. C., T. T., M. W., X. D. and H. C. coordinated the data acquisition (for baseline, resurveys, and long-term follow-up), Y. L., Y. Z. and J. D. contributed to critical revision of the manuscript for important intellectual content and approved the final version of the manuscript. X. W., X. C. and $\mathrm{H}$. Y. were responsible for the overall content of article and data analysis

\section{Competing interests}

The authors declare no competing interests.

\section{Additional information}

Supplementary information is available for this paper at https://doi.org/10.1038/s41598-020-63455-0.

Correspondence and requests for materials should be addressed to Y.Z. or J.D.

Reprints and permissions information is available at www.nature.com/reprints.

Publisher's note Springer Nature remains neutral with regard to jurisdictional claims in published maps and institutional affiliations.

Open Access This article is licensed under a Creative Commons Attribution 4.0 International License, which permits use, sharing, adaptation, distribution and reproduction in any medium or format, as long as you give appropriate credit to the original author(s) and the source, provide a link to the Creative Commons license, and indicate if changes were made. The images or other third party material in this article are included in the article's Creative Commons license, unless indicated otherwise in a credit line to the material. If material is not included in the article's Creative Commons license and your intended use is not permitted by statutory regulation or exceeds the permitted use, you will need to obtain permission directly from the copyright holder. To view a copy of this license, visit http://creativecommons.org/licenses/by/4.0/.

(c) The Author(s) 2020 